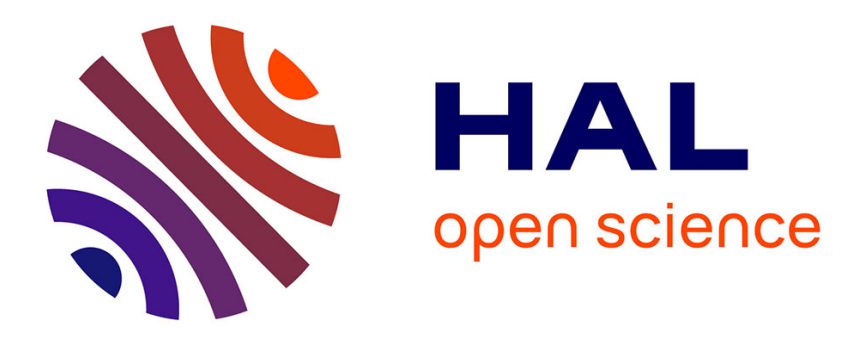

\title{
A Unifying Approach to the Gamma Question
}

Benoit Monin, André Nies

\section{To cite this version:}

Benoit Monin, André Nies. A Unifying Approach to the Gamma Question. LICS 2015, Jul 2015, KYOTO, Japan. pp.585 - 596, 10.1109/LICS.2015.60 . hal-01397264

\section{HAL Id: hal-01397264 \\ https://hal.science/hal-01397264}

Submitted on 17 Nov 2016

HAL is a multi-disciplinary open access archive for the deposit and dissemination of scientific research documents, whether they are published or not. The documents may come from teaching and research institutions in France or abroad, or from public or private research centers.
L'archive ouverte pluridisciplinaire HAL, est destinée au dépôt et à la diffusion de documents scientifiques de niveau recherche, publiés ou non, émanant des établissements d'enseignement et de recherche français ou étrangers, des laboratoires publics ou privés. 


\section{A unifying approach to the Gamma question}

\author{
Benoit Monin \\ Victoria University of Wellington \\ Wellington, New Zealand \\ Email: benoit.monin@computability.fr
}

\author{
André Nies \\ University of Auckland \\ Auckland, New Zealand \\ Email: andre@cs.auckland.ac.nz
}

\begin{abstract}
The Gamma question was formulated by Andrews et al. in "Asymptotic density, computable traceability and 1-randomness" (2013, available at http://www.math.wisc.edu/ $\sim$ lempp/papers/traceable.pdf). It is related to the recent notion of coarse computability which stems from complexity theory. The Gamma value of an oracle set measures to what extent each set computable with the oracle is approximable in the sense of density by a computable set. The closer to 1 this value is, the closer the oracle is to being computable. The Gamma question asks whether this value can be strictly in between 0 and $1 / 2$.

We say that an oracle is weakly Schnorr engulfing if it computes a Schnorr test that succeeds on all computable reals. We show that each non weakly Schnorr engulfing oracle has a Gamma value of at least $1 / 2$. Together with a recent result of Kjos-Hanssen, Stephan, and Terwijn, this establishes new examples of such oracles. We also give a unifying approach to oracles with Gamma value 0 . We say that an oracle is infinitely often equal with bound $h$ if it computes a function that agrees infinitely often with each computable function bounded by $h$. We show that every oracle which is infinitely equal with bound $2^{d^{n}}$ for $d>1$, has a Gamma value of 0 . This provides new examples of such oracles as well.

We present a combinatorial characterization of being weakly Schnorr engulfing via traces, which is inspired by the study of cardinal characteristics in set theory.
\end{abstract}

\section{INTRODUCTION}

Generic-case complexity is a subfield of computational complexity. It started with the observation that some problems that are difficult to solve in full are easy to solve on "most inputs", namely on a set of inputs of density 1 . This notion was introduced by Kapovich et al. [11]. They showed among other things that for a large class of finitely generated groups, the generic case complexity of the word problem is linear.

This notion has recently been extended by Jockusch and Schupp [10]. The authors identify two notions that can be proved to be incomparable. The first is generic computability, where one must always give the right answer, without having to provide an answer for a small set of input. The second is coarse computability, for which one always have to provide an answer, with the right of being wrong on a small set of inputs. In both cases, a set of inputs is considered small if it is of density 0; this will be made precise in the paper.

Then Andrew et al. [1] assign a value $\gamma$ to each set of natural numbers. They use this to assign a value $\Gamma$ to each Turing degree. They prove that the Gamma values of $0,1 / 2$ and 1 can be realized. If a Turing degree has a Gamma value strictly larger than $1 / 2$, then it is computable and its Gamma value in fact equals 1 . They ask whether a Turing degree can have a Gamma value strictly in between 0 and $1 / 2$.

We provide a unifying approach to this question. Among the non-computable degrees, the members of two kinds of degree classes are known to have a Gamma value of $1 / 2$ : the computably traceable degrees, and the computably dominated random degrees. The proofs suggest that in the two cases this holds for very different reasons. We show here that at the contrary, they are both contained in a common class that implies a Gamma value of $1 / 2$ : being not weakly Schnorr engulfing. Together with recent, yet unpublished work of KjosHanssen, Stephan, and Terwijn, this establishes new examples of such degrees.

We also unify the examples of degrees with a Gamma value of 0 , by relating them with a property of degrees we call $H$ infinitely often equal for an appropriate computable bound $H$. We end the paper by giving a combinatorial characterization of being weakly Schnorr engulfing, close to the notion of being $H$-infinitely often equal.

\section{PREliminaries AND NOTATIONS}

In this paper, we work in the space of infinite sequences of 0 's and 1's, the Cantor space, denoted by $2^{\omega}$. We call strings finite sequences of 0 's and 1's, sequences elements of the Cantor space and sets the sets of sequences. We sometimes also use the word sequence to denote sequences of various objects (typically integers), and when we do so we will always specify it to avoid any ambiguity. We also sometimes view a sequence $X$ as the subset of $\omega$ containing $n$ iff $X(n)=1$ without necessarily specify it. For a string $\sigma$, we denote the set of sequences extending $\sigma$ by $[\sigma]$ and we call those sets cylinders. We denote by $\lambda$ the unique probability measure on $2^{\omega}$ such that $\lambda([\sigma])=2^{-|\sigma|}$ for any string $\sigma$, where $|\sigma|$ denotes the length of $\sigma$.

The Cantor space is endowed with the product topology, for which a set is clopen iff it is a finite union of cylinders, and open iff it is a countable union of cylinders. A set $\mathcal{U}$ is effectively open, or $\Sigma_{1}^{0}$, if there exists a computably enumerable sequence of strings $\left\{\sigma_{i}\right\}_{i \in \omega}$ such that $\mathcal{U}=\bigcup_{i}\left[\sigma_{i}\right]$. A set $\mathcal{U}$ is effectively closed, or $\Pi_{1}^{0}$, if it is the complement of a $\Sigma_{1}^{0}$ set. Finally we denote by $\langle n, m\rangle$ a computable bijection from $\omega \times \omega$ to $\omega$.

We now introduce notations which are less standard than those of the previous paragraphs. They will be convenient to expose the work of this paper, especially for results of 
Section V. For a sequence $X \in 2^{\omega}$ and a finite interval $I \subset \omega$, we denote by $X \uparrow_{I}$ the string $X(I(0)) \wedge \ldots \wedge X(I(m-1))$, where $m$ is the length of $I$. For a string $\sigma$ and a finite interval $I$ we write $[\sigma]_{I}$ to denote the set of sequences extending $\sigma$, that is, $\left\{X \in 2^{\omega}: X \uparrow_{I}=\sigma\right\}$. For a finite interval $I \subset \omega$ we write $\mathcal{J} \subseteq 2^{I}$ for a clopen set $\mathcal{J} \subseteq 2^{\omega}$ if there exists a set of strings $\sigma_{0}, \ldots, \sigma_{k}$ of length $|I|$ such that $\mathcal{J}=\bigcup_{i \leq k}\left[\sigma_{i}\right]_{I}$.

In this paper we will be interested in having a canonical coding between sequences and functions $f: \omega \rightarrow \omega$ which are strictly bounded by some $H: \omega \rightarrow \omega$. Such a function $H$ will generally be an order function, that is, a computable function $H$ such that $H(n) \leq H(n+1)$ and $\lim _{n} H(n)=+\infty$. To make the coding work nicely we will consider that the bound $H$ is always of the form $2^{\widetilde{H}(n)}$. Given a sequence $X$ and such a bound $H(n)=2^{\widetilde{H}(n)}$, we denote by $f_{X}$ the function corresponding to $X$. Formally we define $H^{\prime}(n)=\sum_{m<n} \widetilde{H}(m)$ (with $H^{\prime}(n)=0$ ), and $f_{X}(n)$ to be the integer smaller than $2^{\widetilde{H}(n)}$ which is encoded by the string $X \uparrow_{\left[H^{\prime}(n), H^{\prime}(n+1)\right)}$. Conversely, given $f$ with $f(n)<H(n)=2^{\widetilde{H}(n)}$, we write $X_{f}$ to denotes the sequence $X$ such that $f_{X}=f$.

\section{A. Preliminaries on coarse computability}

The notion of coarse computability received quite a lot of recent attention by various authors (see for example [1] and [9]).

Definition II.1. A sequence $A$ is coarsly computable if there is a computable sequence $X$ such that the lim inf of the frequency of positions $n$ on which $A(n)=X(n)$, equals 1 . More formally, let us introduce the function:

$$
\underline{\rho}(Z)=\liminf _{n} \frac{|Z \cap[0, n]|}{n}
$$

The sequence $A$ is coarsly computable if for some computable sequence $X$ we have $\rho(A \leftrightarrow X)=1$, where $A \leftrightarrow X$ denotes the sequence which seen as a subset of $\omega$ contains $n$ iff $A(n)=X(n)$.

A real number can naturally be assigned to non coarsly computable objects. This number can be seen as an indication of how far the object is from being coarsly computable. It is defined by:

$$
\gamma(A)=\sup _{X \text { computable }} \rho(A \leftrightarrow X)
$$

We will refer to this as the gamma value of $A$. Andrews, Cai, Diamondstone, Jockush and Lempp [1] had the interesting idea to define a similar value for Turing degrees, which indicates how far a degree is from being computable:

$$
\Gamma(d)=\inf \left\{\gamma(A): A \in 2^{\omega} \text { is of degree } d\right\}
$$

This will be referred to in this paper as the Gamma value of $d$ (with a capital ' $\mathrm{G}$ '). In practice we will often write $\Gamma(A)$ for a set $A \in 2^{\omega}$ to mean $\Gamma(d)$ where $d$ is the Turing degree of $A$. It is easy to see that one can equivalently consider $\Gamma(A)$ to be the infinium over tha values $\gamma(B)$ for every $B \leq_{T} A$, rather than just every $B \equiv_{T} A$. The reason is that given any $B<_{T} A$, we can add to the sequence $B$ all the information about $A$ at some very sparse computable set of positions, giving a new set Turing equivalent to $A$, with the same gamma value as $B$ 's.

The Gamma question is: which real numbers can be realized by the Gamma value of a degree? In [1] Andrews et al. proved that $\Gamma(A)>1 / 2$ iff $\Gamma(A)=1$ iff $A$ is computable. They also gave examples of sequences $A$ with $\Gamma(A)=1 / 2$ and examples of sequences $A$ with $\Gamma(A)=0$. These examples will be detailed in Section III. It is unkown whether some sequence has a Gamma value strictly between 0 and $1 / 2$.

\section{B. Preliminaries on algorithmic randomness}

Algorithmic randomness uses the tools of computability theory to give a formal definition of the notion of a random infinite binary sequence, a sequence we would expect be the result of independent coin tosses. The reader can refer to [18] or [8] for a background on algorithmic randomness. We briefly discuss the notions important for this paper.

1) Martin-Löf randomness: The first satisfactory definition of randomness was given by Martin-Löf in [16]:

Definition II.2. A Martin-Löf test is given by a uniform intersection $\bigcap_{n} \mathcal{U}_{n}$ of effectively open sets such that the function $n \mapsto \lambda\left(\mathcal{U}_{n}\right)$ is bounded by a decreasing computable function with limit 0 . We say that a sequence $X$ is Martin-Löf random if it belongs to no Martin-Löf test.

Note that one can analogously define sequences which are Martin-Löf random for any computable probability measure $\mu$, by simply replacing $\lambda$ by $\mu$ in the above definition. Such notions will be discussed at the end of Section III-A.

2) Schnorr randomness: The notion of Schnorr randomness was introduced by Schnorr [22], who was aiming at a concept that is more constructive than the one of Martin-Löf.

Definition II.3. A Schnorr test is given by a uniform intersection $\bigcap_{n} \mathcal{U}_{n}$ of effectively open sets such that the function $n \mapsto \lambda\left(\mathcal{U}_{n}\right)$ is computable and decreasing with limit 0 . We say that a sequence $X$ is Schnorr random if it belongs to no Schnorr test.

It is well known that Schnorr randomness is strictly weaker than Martin-Löf randomness. Downey and Griffiths gave in [7] another characterization of Schnorr randomness that can be seen as an effective version of the Borel Cantelli lemma:

Definition II.4. A Schnorr-Solovay test is a computable sequence $\left\{\mathcal{C}_{n}\right\}_{n \in \omega}$ of clopen sets such that $\sum_{n} \lambda\left(\mathcal{C}_{n}\right)$ is finite and computable. A sequence $X$ is captured by a SchnorrSolovay test $\left\{\mathcal{C}_{n}\right\}_{n \in \omega}$ if $X$ is in infinitely many sets $\mathcal{C}_{n}$, that is, $X \in \bigcap_{n} \bigcup_{m \geq n} \mathcal{C}_{m}$.

The term "Schnorr-Solovay test" comes from the analogous notion of "Solovay test", which characterizes Martin-Löf randomness. Downey and Griffiths proved that Schnorr-Solovay 
tests (referred to as total Solovay test in [7]) characterize Schnorr randomness.

Proposition II.5 (Downey/Griffiths [7]). A sequence $X$ is Schnorr random iff it is no captured by any Schnorr-Solovay test.

Definition II.6. A Schnorr-Solovay test $\left\{\mathcal{C}_{n}\right\}_{n \in \omega}$ is called independent if the sequence $\left\{\mathcal{C}_{n}\right\}_{n \in \omega}$ is independent in the usual sense of probability theory: $\lambda\left(\bigcap_{r \in F} \mathcal{C}_{r}\right)=\prod_{r \in F} \lambda\left(\mathcal{C}_{r}\right)$ for each finite set $F$.

As we will see in Section V, each non Schnorr random is captured by an independent Schnorr-Solovay of a very special kind:

Definition II.7. An interval test is given by a uniformly computable sequence of pairs $\left\{I_{n}, \mathcal{J}_{n}\right\}_{n \in \omega}$ where the $I_{n}$ are pairwise disjoint increasing finite intervals (i.e., $\max \left(I_{n}\right)<$ $\min \left(I_{n+1}\right)$ ), each $\mathcal{J}_{n} \subseteq 2^{I_{n}}$ is a clopen set uniformly computable in $n$, and $\sum_{n} \lambda\left(\mathcal{J}_{n}\right)$ is finite and computable.

3) Higher randomness: Randomness has recently been studied from the viewpoint of higher computability. The reader may refer to [21] or [17] for background on higher computability, and to [18] or [17] for background on higher randomness. Here we summarise the main notions used in the paper.

A set of sequences or of integers is $\Pi_{1}^{1}$ if it can be defined by a second order formula of arithmetic where we can have any quantifier over integers, but only universal quantifiers over infinite objects (sequences or functions). A set of sequences or of integers is $\Sigma_{1}^{1}$ if it can be defined by a formula of arithmetic with only existential quantifiers over infinite objects. Finally a set sequences or of integers is $\Delta_{1}^{1}$ if it is both $\Pi_{1}^{1}$ and $\Sigma_{1}^{1}$.

Definition II.8. A sequence $X$ is $\Delta_{1}^{1}$-random if it belongs to no $\Delta_{1}^{1}$ set $\mathcal{A} \subseteq 2^{\omega}$ with $\lambda(\mathcal{A})=0$. A sequence $X$ is $\Pi_{1}^{1}$ random if it belongs to no $\Pi_{1}^{1}$ set $\mathcal{A} \subseteq 2^{\omega}$ with $\lambda(\mathcal{A})=0$.

The Gandy-Spector theorem gives a powerful analogy between the notions of computable/recursively enumerable sets of integers and $\Delta_{1}^{1} / \Pi_{1}^{1}$ sets of integers. Informally one can picture a $\Pi_{1}^{1}$ set of integers as being effectively enumerable, but along some "ordinal stages of computation" $\alpha<\omega_{1}^{c k}$ rather than just finite stages of computation, where $\omega_{1}^{c k}$ is defined as the smallest non computable ordinal, that is, the smallest ordinal $\alpha$ such that there is no c.e. relation $R \subseteq \omega \times \omega$ which is a well order of order-type $\alpha$. Similarly, one can informally picture a $\Delta_{1}^{1}$ set of integers as being effectively enumerable along some "ordinal stages of computation", but bounded by some $\alpha<\omega_{1}^{c k}$.

One set of particular interest in higher randomness is the set of sequences $X$ such that the smallest non-computable ordinal relatively to $X$, namely $\omega_{1}^{X}$, is larger than $\omega_{1}^{c k}:\left\{X \in 2^{\omega}\right.$ : $\left.\omega_{1}^{X}>\omega_{1}^{c k}\right\}$. We have the following theorem:

Theorem II.9 (Chong, Nies, Yu [4]). A sequence $X$ is $\Pi_{1}^{1}$ random iff it is $\Delta_{1}^{1}$-random and $\omega_{1}^{X}=\omega_{1}^{c k}$.
There exists in higher computability a version of the halting problem. This is the set called Kleene's $\mathcal{O}$, defined as the set of all the codes $e$ such that the binary c.e. relation coded by $e$ is a total well-order of the integers ${ }^{1}$. Every $\Pi_{1}^{1}$ set is many-one reducible to Kleene's $\mathcal{O}$.

Theorem II.10. For a sequence $X$ we have $\omega_{1}^{X}>\omega_{1}^{c k}$ iff Klenne's $\mathcal{O}$ is $\Delta_{1}^{1}(X)$.

Finally we state here what is known as the van Lambalgen theorem for $\Pi_{1}^{1}$-randomness. The sequence $X \oplus Y$ is defined by $(X \oplus Y)(2 n)=X(n)$ and $(X \oplus Y)(2 n+1)=Y(n)$.

Theorem II.11. For sequences $X, Y$ we have that $X \oplus Y$ is $\Pi_{1}^{1}$-random iff $X$ is $\Pi_{1}^{1}$-random and $Y$ is $\Pi_{1}^{1}(X)$-random.

In particular, if a $\Pi_{1}^{1}$-random sequence $X$ is in a $\Pi_{1}^{1}(Y)$ nullset then $Y$ is in a $\Pi_{1}^{1}(X)$ nullset.

\section{Preliminaries on weakly Schnorr engulfing and traces}

It is well known that unlike the case of Martin-Löf randomness, there exists no universal Schnorr test, that is, no Schnorr test covering all the others. One can prove this by showing that every $\Pi_{1}^{0}$ set of computable positive measure contains a computable sequence, so that no Schnorr test contains every computable sequence (see for instance Fact 3.5.9 in [18]). We are interested in oracles that strengthen the power of Schnorr tests, in that some Schnorr test relative to the oracle captures all the computable sequences.

Definition II.12. A sequence $A$ is weakly Schnorr engulfing if there exists an $A$-Schnorr test containing all the computable sequences.

A weaker property of oracles has already been proved equivalent to a tracing property: some Schnorr test relative to the oracle is not covered by any plain Schnorr test. We first define the tracing property.

Definition II.13 (Terwijn and Zambella [23]). A computable trace is a uniform sequence $\left\{T_{n}\right\}_{n \in \omega}$ of finite sets of integers given by strong indices. Formally $T_{n}=D_{p(n)}$ where $p: \omega \rightarrow$ $\omega$ is a computable function and $D_{n}$ is the set containing $m$ iff there is a 1 at position $m$ of the binary encoding of $n$. An oracle $A$ is computably traceable if there exists a computable bound $H: \omega \rightarrow \omega$ such that for every function $f \leq_{T} A$, there exists a computable trace $\left\{T_{n}\right\}_{n \in \omega}$ such that $\left|T_{n}\right|<H(n)$ and such that $f(n) \in T_{n}$ for every $n$.

Intuitively, an oracle is computably traceable if every function $f$ it computes is 'close to computable', in that one can compute a small set of values $f(n)$ belongs to. By a result Terwijn and Zambella [23] together with Kjos-Hanssen, Nies and Stephan [13] we have:

Theorem II.14. For an oracle $A$ the following are equivalent: - A is not computably traceable.

${ }^{1}$ The definition of Kleene is actually more complex in order to ease effective definitions by induction over elements of this set. For our purpose the above definition is enough. 
- There is an A-Schnorr test covered by no Schnorr test.

- There is an A-Schnorr test containing a Schnorr random.

Oracles failing any of these properties are said to be low for Schnorr randomness. We will show the connection between being weak Schnorr engulfing and the Gamma question. We shall also give in Section $\mathrm{V}$ a combinatorial characterization of being weakly Schnorr engulfing, by showing its equivalence with another tracing property.

\section{Preliminaries on hyperimmune sequences}

The following is central notion of computability theory.

Definition II.15. An oracle $X$ is computably dominated if for every function $f \leq_{T} X$, there exists a computable function $g$ such that $f \leq g$, that is, $f(n) \leq g(n)$ for every $n$. An oracle $X$ is of hyperimmune degree, or simply hyperimmune if it is not computably dominated.

Kurtz proved in [15] that a sequence is hyperimmune iff it Turing computes a weakly-1-generic sequence, that is, a sequence which is in every dense $\Sigma_{1}^{0}$ subset of $2^{\omega}$. We state here a less famous, though interesting, third equivalent notion:

Definition II.16. A function $f: \omega \mapsto \omega$ if infinitely often equal (or i.o.e.) if it is equal infinitely often to every computable function. A sequence $A$ is of i.o.e. degree if it Turing computes an i.o.e. function.

For completeness' sake we include a proof of the wellknown equivalence between being of hyperimmune degree and being of i.o.e. degree:

Proposition II.17. For a sequence $A$ the following are equivalent:

- A is of hyperimmune degree.

- $A$ is of i.o.e degree.

Proof. If $f: \omega \mapsto \omega$ is an i.o.e. function, then $f+1$ is clearly dominated by no computable function. Conversely suppose that $g: \omega \mapsto \omega$ is a function bounded by no computable function. Let $\left\{\Phi_{e}\right\}_{e \in \omega}$ be an effective list of the partial computable functions and $\left\{R_{e}\right\}_{e \in \omega}$ a list of Boolean values initialized to false. At stage $n$, we define $f(n)$ to be $\Phi_{k}(n)[g(n)]$ where $k$ is the least integer smaller than $n$ such that $\Phi_{k}(n)[g(n)]$ halts and such that $R_{k}$ is false (if no such $k$ exists we let $f(n)=0)$. Then set $R_{k}$ to true. Note first that a function $f$ is i.o.e. iff it is equal once to every computable function (using the finite-change closure property of the set of computable functions). If $\Phi_{e}$ is total then the function $n \mapsto \min \left\{t: \Phi_{e}(n)[t]\right.$ halts $\}$ is total and computable, thus it is dominated by $g$ infinitely often. It is easily to check now that $f$ is equal at least once to every computable function.

In this paper, we will be interested in hyperimmune sequences through their i.o.e. characterization. We will also be interested in computably dominated sequences that are random. It is well known that while the set of computably dominated elements has measure 0 , some of them are still Schnorr random. It is also well known that each computably dominated Schnorr random is actually Martin-Löf random. So we will refer to them simply as the computably dominated randoms.

\section{THE GAMMA VALUES}

\section{A. Gamma value of $1 / 2$ and being weakly Schnorr engulfing}

The only two known examples of sequences with a Gamma value of $1 / 2$ are the (non-computable ) computably traceable sequences and the computably dominated random sequences [1]. They are quite different from each other: While the sequences of first kind are close to computable, the sequences of second kind are far from computable (as they are random).

We identify here a third property of an oracle implied by both, which suffices to get a gamma value of $1 / 2$ : being not weakly Schnorr engulfing. As computably traceable sequences are low for Schnorr randomness, they are not weakly Schnorr engulfing (see Theorem II.14). Rupprecht [20] proved that computably dominated random sequences are not weakly Schnorr engulfing. It remains to prove that any non weakly Schnorr engulfing sequences has a gamma value of $1 / 2$ (or of 1 in case it is computable).

Theorem III.1. Let $A$ be not weakly Schnorr engulfing. Then $\Gamma(A) \geq 1 / 2$.

Proof. Let $d \in \mathbb{N}$. For each $B \leq_{\mathrm{T}} A$ we will define a SchnorrSolovay test $\left(G_{k}\right)_{k \in \mathbb{N}}$ relative to $A$ such that $\rho(B \leftrightarrow R) \geq$ $1 / 2-1 / d$ for each sequence $R$ not captured by this test. For each $d$ some computable sequence $R$ passes the test, so this will show that $\gamma(B) \geq 1 / 2$.

Let $I_{k}$ be defined inductively as the set of $k$ consecutive integers following $I_{k-1}: I_{0}=\emptyset, I_{1}=\{1\}, I_{2}=\{2,3\}, \ldots$ Given $k$ let

$$
\begin{gathered}
G_{k}=\left\{Z: Z(i) \neq B(i) \text { for a ratio of bits in } I_{k}\right. \text { of at least } \\
1 / 2+1 / d\}
\end{gathered}
$$

which is a clopen set computed uniformly in $k$ from $A$. By the usual Chernoff bounds we have $\lambda G_{k} \leq e^{-\frac{2 k}{d^{2}}}$. Clearly $\int_{r}^{\infty} e^{-\frac{2 x}{d^{2}}} d x \geq \sum_{k=r+1}^{\infty} e^{-\frac{2 k}{d^{2}}}$. Since $\int_{r}^{\infty} e^{-\frac{2 x}{d^{2}}} d x=d^{2} e^{-\frac{2 r}{d^{2}}}$ effectively converges to 0 as $r \rightarrow \infty$, the real $\sum_{k} \lambda G_{k}$ is computable in $A$. Thus $\left(G_{k}\right)_{k \in \mathbb{N}}$ is a Schnorr-Solovay test relative to $A$ as required.

In [1] it is proved that any sequence which is computably dominated and random with respect to a computable measure has a Gamma value of $1 / 2$ (unless it is computable, which can happen if it is an atom of the measure). Rupprecht's proof in [20] can be modified to show that no sequence which is computably dominated and random for a computable measure is weakly Schnorr engulfing. It is natural to wonder if there are other sequences with Gamma values of $1 / 2$. In very recent work related to the authors through personal communication, Kjos-Hanssen, Stephan, and Terwijn constructed a sequence which is not weakly Schnorr engulfing, not computably dominated, and not DNC (see e.g. [18, Ch. 4] for the definition of DNC, which abbreviates "diagonally non-computable"). 
A non-DNC sequence cannot be random for any atomless computable measure. (For instance, use Demuth's result [6] that any Martin-Löf random with respect to an atomless computable measure Turing computes a Martin-Löf random with respect to $\lambda$, together with the fact that every MartinLöf random is DNC.) Thus, together with the result of KjosHanssen et al., we have obtained new examples of sequences with a Gamma value of $1 / 2$.

\section{B. Gamma value of 0 and infinite equality}

The two known examples of sequences with a Gamma value of 0 are the sequences of hyperimmune degree, and the sequences of PA degree. These latter are the sequences which Turing compute a complete extension of Peano arithmetic. It is well known that they are also the sequences which Turing compute a member of any non-empty $\Pi_{1}^{0}$ set, uniformly in a code for this set. It is also well known that some of them are computably dominated. These two types of sequences seem to have a Gamma value of 0 for quite different reasons, when one looks at the respective proofs in [1]. We identify here a third notion implied by both, which already suffices to get a Gamma value of 0 . This notion is a weakening of being i.o.e., where one introduces a bound on the functions.

Definition III.2. Given a computable bound $H: \omega \mapsto \omega$ we say that $f: \omega \mapsto \omega$ is $H$-infinitely often equal (or $H$-i.o.e.) if $f$ equals infinitely often every computable function strictly bounded by $H$. A sequence $A$ is of $H$-i.o.e. degree if $A$ Turing computes an $H$-i.o.e. function.

Recall that Proposition II.17 showed that sequences of hyperimmune degree are of i.o.e. degree (with no bound).

Theorem III.3. Any PA degree is $H$-i.o.e. for any computable bound $H$ of the form $2^{\widetilde{H}}$.

Proof. For any $A$ of PA degree, there exists an $A$-computable list $\left\{X_{e}\right\}_{e \in \omega}$ of sequences that contains all the computable ones. To see that, recall that $A$ is of PA degree iff $A$ uniformly Turing computes a member of any non-empty $\Pi_{1}^{0}$ set. Apply that uniformly in a partial computable function $\Phi$ to the $\Pi_{1}^{0}$ set

$$
\left\{X: \forall n \forall t\left[\Phi_{e}(n)[t] \uparrow \text { or } \Phi_{e}(n)[t]=X(n)\right]\right\} \text {. }
$$

Now given such a list and any total computable function $\widetilde{H}$, define $H^{\prime}(n)=\sum_{m<n} \widetilde{H}(m)$ (with $H^{\prime}(n)=0$ ). We then simply define the $A$-computable function $f$ which to $n$ associates the natual number corresponding to the string $X_{n} \uparrow_{\left[H^{\prime}(n), H^{\prime}(n+1)\right)}$. If is clear that $f$ is $H$-i.o.e. where $H(n)=2^{\widetilde{H}(n)}$.

We now prove that being of $H$-i.o.e. degree for $H$ sufficiently fast growing implies having a Gamma value of 0 .

Theorem III.4. Let $d>1$ be a real. If $A$ is of $2^{\left(d^{n}\right)}$-i.o.e. degree, then $\Gamma(A)=0$.

Proof. We first prove that if $A$ is of $2^{\left(a^{n}\right)}$-i.o.e. degree for a natural number $a>1$, then $\gamma(A) \leq 1 / a$. Consider a $2^{\left(a^{n}\right)}$. i.o.e. function $f$ that we can bound without loss of generality by $2^{\left(a^{n}\right)}$. Let $H(n)=\sum_{m<n} a^{m}$ (with $H(n)=0$ ). We define the $f$-computable set $B_{f}$ such that $B_{f}\left\lceil_{[H(n), H(n+1))}\right.$ is equal to the string corresponding to the $n$-th value of $f$. Consider now any computable sequence $X$ and its bitwise complement $\bar{X}$, together with the function $f_{\bar{X}}$ which to $n$ associates

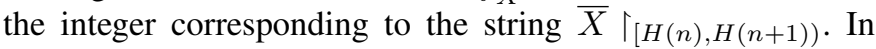
particular as $f$ is infinitely often equal to $f_{\bar{X}}$, there are infinitely many $n$ such that from position $H(n)$ to $H(n+1)-1$, sequences $X$ and $B$ disagree all the time, which implies

$$
\frac{|(B \leftrightarrow X) \cap[0, H(n+1))|}{H(n+1)} \leq \frac{H(n)}{H(n+1)}
$$

And as we have $a^{n}=(a-1) H(n)-1$ we have $H(n+1)=$ $H(n)+(a-1) H(n)-1$ and we then have a ratio of at most $H(n) /(a H(n)-1)$ bits which are guessed correctly by $X$ on the initial segment of length $H(n+1)$. As this happens infinitely often and for every computable sequence $X$, we then have $\gamma(B) \leq 1 / a$ and hence $\gamma(A) \leq 1 / a$.

We shall now prove that if $A$ is of $2^{\left(d^{n}\right)}$-i.o.e. degree for any real $d>1$, then $A$ is of $2^{\left(a^{n}\right)}$-degree for any natural number $a>1$. To do so we first argue that if $f$ is $H$-i.o.e. for some function $H$, then the function $n \mapsto f(2 n)$ is $H(2 n)$ i.o.e. or the function $n \mapsto f(2 n+1)$ is $H(2 n+1)$-i.o.e. Indeed suppose that some computable function $g_{1}$ bounded by $H(2 n)$ is such that $g_{1}(n) \neq f(2 n)$ for every $n$ and that some computable function $g_{2}$ bounded by $H(2+1)$ is such that $g_{2}(n) \neq f(2 n+1)$ for every $n$. Then the computable function $g$ such that $g(2 n)=g_{1}(n)$ and $g(2 n+1)=g_{2}(n)$ is never equal to $f$, which is a contradiction.

Given a $2^{\left(d^{n}\right)}$-i.o.e. function $f$ and any integer $a>1$, let $k$ be such that $2^{\left(d^{k \times n}\right)} \geq 2^{\left(a^{n}\right)}$ (a value $k$ bigger than $\log _{d}(a)$ suffices). By repeating the operation described above sufficiently often, we easily see how to compute from $f$ a function $f^{\prime}$ which is $2^{\left(d^{k \times n}\right)}$-i.o.e. and hence $2^{\left(a^{n}\right)}$-i.o.e. It follows that $\Gamma(A)=0$.

Rupprecht [20] constructed a sequence which is weakly Schnorr engulfing, computably dominated and not DNC. His proof can be slightly modified to construct a sequence which is for any given computable function $\widetilde{H}$, of $2^{\widetilde{H}(n)}$-i.o.e. degree, and both computably dominated and not DNC. As every PA degree is DNC, this provides new examples of sequences with a Gamma value of 0 .

\section{Gamma values with respect to bases other than 2}

We consider here the Gamma value for real numbers expressed in different bases. For an integer $b \geq 2$ we denote the space of infinite sequences of elements in $\{0, \ldots, b-1\}$ by $b^{\omega}$. For $A \in b^{\omega}$ we define the value $\gamma_{b}(A)$ as before, except we now consider a supremum over computable elements of $b^{\omega}$. The definition of $\Gamma_{b}(A)$ is also as before, except we now consider an infimum over elements of $b^{\omega}$ which are Turing equivalent to $A$. Finally for a (non rational) real $r \in \mathbb{R}$ we define $\Gamma_{b}(r)$ to be $\Gamma_{b}(A)$ for $A \in b^{\omega}$ the canonical representation of $r$ in base $b$.

Let us argue that for any base $b \geq 2$ and any real $r$ we have $\Gamma_{b+1}(r) \leq \Gamma_{b}(r)$. Indeed for every sequence $A \in b^{\omega}$ we 
obviously have $\gamma_{b}(A)=\gamma_{b+1}(A)$. As every elements of $b^{\omega}$ is also an element of $(b+1)^{\omega}$, the infimum in the definition of $\Gamma_{b+1}(r)$ is done over more elements than in the definition of $\Gamma_{b}(r)$. In particular if $\gamma_{2}(r)=0$ then $\gamma_{b}(r)=0$ for any $b \geq 2$.

By straightforward modifications of the proof of Theorem III.1, any sequence $A \in b^{\omega}$ which is not weakly Schnorr engulfing has a Gamma value of $1 / b$. What is really of interest here is the proof that any sequence $A \in b^{\omega}$ such that $\Gamma_{b}(A)>1 / b$ is computable: The proof of this in [1] for the case $b=2$ uses a "majority vote" technique, that cannot be used directly for larger bases. This will be made clear in what follows.

Definition III.5. For any sequence $A \in 2^{\omega}$, seen as a subset of $\omega$, we denote by $\#_{c}^{A}: \omega^{c} \rightarrow \omega$ the function which on $x_{1}, \ldots, x_{c}$ returns $\left|A \cap\left\{x_{1}, \ldots, x_{c}\right\}\right|$.

Note that $\#_{c}^{A}$ can take at most $c+1$ distinct values. Kummer [14] proved that if $A$ is not computable, one cannot trace $\#_{c}^{A}$ by a c.e. trace containing strictly less than $c+1$ values. The proof was later simplified by Owings [19]:

Theorem III.6. [Kummer] Let $c \geq 2$. Suppose $A$ is an oracle such that $\#_{c}^{A}$ is traceable via some trace $\left\{T_{n}\right\}_{n \in \omega}$, where each $T_{n}$ is c.e. uniformly in $n$ and $\left|T_{n}\right| \leq c$. Then $A$ is computable.

We will use this in the proof of the following theorem:

Theorem III.7. Let $A \in b^{\omega}$. If $\Gamma_{b}(A)>1 / b$ then $A$ is computable.

Proof. Let $\widetilde{A} \in 2^{\omega}$ be some binary encoding of $A$. The sequence $\#_{b-1}^{\widetilde{A}}$ belongs to $b^{\omega}$. We perform a majority vote trick as in [1], except that we now do not need an absolute majority to win. Consider a sequence $B \in b^{\omega}$ which encodes each bit of $\#_{b-1}^{\widetilde{A}}$ with many repetitions. Formally, we define inductively intervals $I_{n}$ by $I_{0}=\{0\}$ and $I_{n+1}=\{k$ : $\left.a_{n}<k \leq(n+1) \times s_{n}\right\}$ where $a_{n}$ is the last position in the interval $I_{n}$ and $s_{n}$ the sum of the length of the intervals $I_{0}$ to $I_{n}$. For any $k \in I_{n}$ we define $B(k)=\#_{b-1}^{\widetilde{A}}(n)$. As $B$ is Turing computable from $A$, we must have $\gamma_{b}(B)>1 / b$, there is then a computable sequence $C$ such that $\lim _{n \rightarrow} \mid B \leftrightarrow$ $C \cap[0, n] \mid / n>1 / b$. We now claim that for any $n$ large enough, the percentage of positions $k$ in $I_{n}$ such that $C(k)=\#_{b-1}^{\widetilde{A}}(n)$ is strictly greater than $1 / b$. Suppose otherwise. In particular, for arbitrarily large $n$ we have less than or equal to $1 / b$ positions in $I_{n}$ which are guessed correctly by $C$. Recall $s_{n-1}$ is the number of positions before $I_{n}$. As there are $n$ more positions in $I_{n}$ than in all the previous intervals together, we have at most

$$
\frac{s_{n-1}+\left(n s_{n-1}\right) / b}{s_{n-1}+n s_{n-1}}=\frac{1+n / b}{1+n}
$$

many positions guessed correctly. This expression has limit $1 / b$ as $n$ goes to infinity. It then follows that

$$
\liminf _{n}|B \leftrightarrow C \cap[0, n]| / n \leq 1 / b,
$$

which is a contradiction.
Therefore for $n$ large enough, the sequence $C$ must guess in the interval $I_{n}$ strictly more than $1 / b$ of the bits correctly. Also they can be at most $b-1$ values which are given by $C$ with a ratio strictly bigger than $1 / b$. By building the computable trace with all these values, we have a trace for $\#_{b-1}^{\widetilde{A}}$, which implies by Theorem III.6 that $\widetilde{A}$, and then $A$, is computable.

Question III.8. Let $r \in[0,1]$ be non-computable. Do we have for all integers $b, c \geq 2$ that $\Gamma_{b}(r)=1 / b$ iff $\Gamma_{c}(r)=1 / c$ ?

\section{The Gamma value in the higher setting}

In this section we study a notion analogous to being weakly Schnorr engulfing in the setting of higher computability. Thereafter we discuss a higher version of the Gamma question.

Definition III.9. A sequence $A$ is weakly $\Delta_{1}^{1}$ engulfing if there is a $\Delta_{1}^{1}(A)$ nullset containing every $\Delta_{1}^{1}$ sequence.

For an $A$-computable ordinal $\alpha$, we write $A^{(\alpha)}$ to denote the $\alpha$-th jump of $A$. There are several equivalent ways to concretely define this set, for example via $H$-sets, as initially done by Kleene and Spector (see [21]), or as the set of codes for $A$-c.e. binary relations coding for total orders of order-type strictly smaller than some ordinal, as in [17]. The important point is that $A^{(\alpha)}$ should be a $\Sigma_{\alpha}^{0}(A)$-complete set.

It is well known (see for example [5] or Proposition 4.2.5 in [17]) that if $A$ is $\Delta_{1}^{1}$-random, then it is $G L_{\alpha}$ for every computable ordinal $\alpha$, that is, $A^{(\alpha)} \equiv_{T} A \oplus \emptyset^{(\alpha)}$. Using this, we prove the following theorem about $\Pi_{1}^{1}$-random sequences:

Theorem III.10. Suppose $A$ is $\Pi_{1}^{1}$ random. Then $A$ is not weakly $\Delta_{1}^{1}$ engulfing.

Proof. Consider a $\Delta_{1}^{1}(A)$ nullset $\mathcal{S}$. As $\omega_{1}^{A}=\omega_{1}^{c k}$ we have some computable ordinal $\alpha$ such that $\mathcal{S}$ is a $\Sigma_{\alpha}^{0}(A)$ set. By the effective regularity of Lebesgue measure relativized to $A$, we can approximate $\mathcal{S}$ from above by a uniform intersection of $\Delta_{1}^{1}(A)$ open sets $\bigcap_{n} \mathcal{U}_{n}$ with $\lambda\left(\mathcal{U}_{n}\right) \leq 2^{-n}$, and still because $\omega_{1}^{A}=\omega_{1}^{c k}$ we have a computable ordinal $\alpha$ such that each $\mathcal{U}_{n}$ is $\Sigma_{1}^{0}\left(A^{(\alpha)}\right)$ uniformly in $n$. Now because $A$ is $\Delta_{1}^{1}$ random it is $G L_{\alpha}$ and then each $\mathcal{U}_{n}$ is also $\Sigma_{1}^{0}\left(A \oplus \emptyset^{(\alpha)}\right)$ uniformly in $n$. Let $e$ be an index for the $\Pi_{2}^{0}\left(A \oplus \emptyset^{(\alpha)}\right)$ set $\bigcap_{n} \mathcal{U}_{n}$. We can now transform $e$ such that for any oracle $X$ we have $\lambda\left(\mathcal{U}_{n}^{X}\right) \leq 2^{-n}$, without changing $\mathcal{U}_{n}$ on oracle for which we already have $\lambda\left(\mathcal{U}_{n}^{X}\right) \leq 2^{-n}$ (in particular on the oracle $\left.A \oplus \emptyset^{(\alpha)}\right)$. For any $X$ consider now the $\Delta_{1}^{1}(X)$ set:

$$
\mathcal{N}_{X}=\left\{Z: X \in \bigcap_{n} \mathcal{U}_{n}^{Z \oplus \emptyset^{(\alpha)}}\right\}
$$

and consider the $\Delta_{1}^{1}$ set:

$$
\mathcal{N}=\left\{X: \lambda\left(\mathcal{N}_{X}\right)>0\right\}
$$

We claim that $\mathcal{N}$ contains every $\Delta_{1}^{1}$ sequence belonging to $\bigcap_{n} \mathcal{U}_{n}^{A \oplus \emptyset^{(\alpha)}}$ and that $\lambda(\mathcal{N})=0$. If $X$ is $\Delta_{1}^{1}$ and belongs to $\bigcap_{n} \mathcal{U}_{n}^{A \oplus \emptyset^{(\alpha)}}$ we clearly have $A \in \mathcal{N}_{X}$. But as $\mathcal{N}_{X}$ is $\Delta_{1}^{1}$ and as $A$ is $\Delta_{1}^{1}$ random, we must have $\lambda\left(\mathcal{N}_{X}\right)>0$. Thus every $\Delta_{1}^{1}$ sequence contained in $\bigcap_{n} \mathcal{U}_{n}^{A \oplus \emptyset^{(\alpha)}}$ is also contained in $\mathcal{N}$. 
To prove $\lambda(\mathcal{N})=0$ it suffices to prove that $\mathcal{N}$ contains no $\Pi_{1}^{1}$ random sequence. This is clear using the van Lambalgen theorem for $\Pi_{1}^{1}$-randomness (Theorem II.11). Suppose that $Y$ is $\Pi_{1}^{1}$-random in order to prove $Y \notin \mathcal{N}$. Suppose for some $Z$ we have $Y \in \bigcap_{n} \mathcal{U}_{n}^{Z \oplus \emptyset^{(\alpha)}}$. In particular as $Y$ is not $\Pi_{1}^{1}(Z)$ random $Z$ is not $\Pi_{1}^{1}(Y)$-random. Then $\mathcal{N}_{Y}$ is included in the set of sequences which are not $\Pi_{1}^{1}(Y)$-random. As this is a set of measure 0 we have $\lambda\left(\mathcal{N}_{Y}\right)=0$. Hence $Y$ is not in $\mathcal{N}$.

We now define counterparts of $\gamma$ and $\Gamma$ in the higher setting:

$$
\begin{gathered}
\gamma_{h}(A)=\sup _{X \in \Delta_{1}^{1}} \underline{\rho}(A \leftrightarrow X) \\
\Gamma_{h}(A)=\inf \left\{\gamma_{h}(B): B \text { is } \Delta_{1}^{1}(A)\right\}
\end{gathered}
$$

As in the setting of computability, $\Gamma_{h}(A)>1 / 2$ iff $A$ is $\Delta_{1}^{1}$. To show this, one follows the same proof as the one of Proposition 1.4. in [1], using a "majority vote" technique.

Still as in the setting of computability, and following the same proof as the one of Theorem III.1, if $A$ is not weakly $\Delta_{1}^{1}$ engulfing then $\Gamma_{h}(A)=1 / 2$. In particular:

Theorem III.11. Every $\Pi_{1}^{1}$-random sequence $Z$ satisfies $\Gamma_{h}(Z)=1 / 2$.

Consequently, for $\Gamma$, the situation is quite different in the higher setting: The set of sequences with a Gamma value of $1 / 2$ has measure 1 , whereas it has measure 0 in the lower setting.

A notion of being infinitely often equal also makes sense in the higher setting.

Definition III.12. Let $H: \omega \mapsto \omega$ be a $\Delta_{1}^{1}$ bound. A function $f: \omega \mapsto \omega$ is higher $H$-i.o.e. if it is infinitely often equal to every $\Delta_{1}^{1}$ function bounded by $H$. A sequence $A$ is of $H$-i.o.e. hyperdegree if there is some higher $H$-i.o.e. function which is $\Delta_{1}^{1}(A)$.

It is clear that the proof of Theorem III.4 can be adapted to the higher setting to obtain for any real $d>1$ that any $A$ of $2^{d^{n}}$-i.o.e. hyperdegree has a higher Gamma value of 0 . In particular, if $\omega_{1}^{X}>\omega_{1}^{c k}$ then $\Gamma_{h}(X)=0$.

A difference with the lower setting is that we don't have an analogue of the fact that every hyperimmune function computes an infinitely often equal function (see II.17). Kihara [12] has shown that there is a sequence $X$ such that there exists a $\Delta_{1}^{1}(X)$ function $f$ bounded by no $\Delta_{1}^{1}$ function, but such that there exists no $\Delta_{1}^{1}(X)$ infinitely often equal function.

\section{INFINITE EQUALITY}

We have seen that both being weakly Schnorr engulfing and the Gamma question are connected to the notion of infinite equality. We study this notion on its own right in some more detail.

It is clear that the two implications of Fig. 1 hold. We should also argue that they are strict. First let us prove the left part of Fig. 1. It is obvious that being of 2 -i.o.e. degree is equivalent to being computable, but for $c>2$ some more complicated

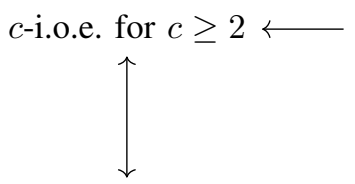

not computable
$H(n)$-i.o.e with $H$ computable order function s.t. $\sum_{n} \frac{1}{H(n)}=\infty$

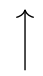

$H(n)$-i.o.e with $H$ computable order function s.t. $\sum_{n} \frac{1}{H(n)}<\infty$

Fig. 1. Known implications for i.o.e. degrees

argument is required. Here again, we can use the function $\#_{c}^{A}$ of Definition III.5 and Theorem III.6 to deduce the following:

Theorem IV.1. Suppose $A$ is not c-i.o.e. for some integer $c \geq 2$. Then $A$ is computable.

Proof. The function $g\left(\left\langle x_{1}, \ldots, x_{c-1}\right\rangle\right)=\#_{c}^{A}\left(x_{1}, \ldots, x_{c-1}\right)$ is clearly $A$-computable and always strictly less than $c$. Suppose it is always different from some computable function $f$ strictly bounded by $c$. Then we can capture $g$ (and hence $\#_{c}^{A}$ ) with the computable trace $\left\{T_{n}\right\}_{n \in \omega}$ such that $T_{n}$ contains every value less than $c$ different from $f(n)$. It follows from Theorem III.6 that $A$ is computable.

By the following proposition, the first implication is strict: there exists a non-computable sequence which is not of $\mathrm{H}$ i.o.e. degree for any order function $H$.

Proposition IV.2. Suppose A computably traceable. For any order function $H$ and any function $f \leq_{T} A$, there exists a computable function $g<H$ such that $f$ is always different from $g$.

Proof. Suppose $f<H$ is Turing below some computably traceable set $A$. As proved in [23], for any computable order function $H^{\prime}$, as slowly growing as we want, the function $f$ can be traced via a computable trace $\left\{T_{n}\right\}_{n \in \omega}$ bounded by $H^{\prime}$. We simply take $H^{\prime}<H$. To compute $g(n)$ we can then take any value smaller than $H(n)$ and not in $T_{n}$.

Let us now prove that the second implication is strict: for any order function $H$ there exists a sequence of $H(n)$-i.o.e. degree such that $\sum_{n} 1 / H(n)=\infty$, but not of $H(n)$-i.o.e. degree for any order function $H$ such that $\sum_{n} 1 / H(n)<\infty$. This is done via a computably dominated random. For the next proposition, we use the very weak notion of Kurtz randomness, which is defined by not being in any $\Pi_{1}^{0}$ set of measure 0 .

Proposition IV.3. Suppose A Kurtz random. Then $A$ is of $H(n)$-i.o.e. degree for any order function $H=2^{\widetilde{H}}$ such that $\sum_{n} 1 / H(n)=\infty$.

Proof. Consider a computable function $f<H$. Let $H^{\prime}(n)=$ $\sum_{m<n} \widetilde{H}(m)\left(\right.$ with $\left.H^{\prime}(0)=0\right)$. Let $I_{n}=\left[H^{\prime}(n), H^{\prime}(n+1)\right)$, then we have by hypothesis that $\sum_{n} \lambda\left([f(n)]_{I_{n}}\right)=\infty$ (where 
$f(n)$ is seen as a string of length $I_{n}$ ). Also as the $I_{n}$ are pariwise disjoint, by Borel-Cantelli we have

$$
\lambda\left(\bigcap_{n} \bigcup_{m \geq n}[f(m)]_{I_{m}}\right)=1
$$

and then for each $n$ we have $\lambda\left(\bigcup_{m \geq n}[f(m)]_{I_{m}}\right)=1$. It follows that if $X$ is Kurtz random there are infintely many $n$ such that $X \uparrow_{I_{n}}=f(n)$, where $X \uparrow_{I_{n}}$ is seen as an integer smaller than $2^{\left|I_{n}\right|}$. Also this is true for any computable function $f$, and it then follows that the $X$-computable function $g(n)=X \uparrow_{I_{n}}$ is $H(n)$-i.o.e.

It is easy to prove that if a sequence is of $H(n)$-i.o.e. degree for an order function $H$ such that $\sum_{n} 1 / H(n)<\infty$, then it is weakly Schnorr engulfing. A more general statement will actually be proved in the next section (see Proposition V.3). It follows that any computably dominated random is of $H(n)$ i.o.e. degree for any order function $H$ such that $\sum_{n} 1 / H(n)=$ $\infty$, but not of $H(n)$-i.o.e. degree for any computable order function $H$ such that $\sum_{n} 1 / H(n)<\infty$. For instance, such a sequence is of $2^{\log (n)}$-i.o.e. degree and not of $2^{2 \log (n)}$-i.o.e. degree.

Question IV.4. Is there some $X$ of $2^{n}$-i.o.e degree that is not of $f(n)$-i.o.e degree for some computable $f(n)>>2^{n}$ ? If yes, can $f(n)$ be taken to be $2^{2^{n}}$ ?

\section{WEAKLY SCHNORR ENGULFING AND TRACING}

The notions of being weakly Schnorr engulfing and being $H$-infinitely often equal for $H$ sufficiently fast growing are both related to the Gamma question. At first sight these notions seems unrelated. We give here a combinatorial characterization of being weakly Schnorr engulfing, using traces whose members are bounded by some computable function. This will bring it closer to the notion of being of $H$-i.o.e. degree for some $H$. We note that both infinitely equal and the tracing property that turns out to be equivalent to weakly Schnorr engulfing were introduced in search of computability theoretic analogues of similar combinatorial notions from set theory used to analyse cardinal characteristics (see [3]).

Definition V.1. For a computable function $H: \omega \mapsto \omega$ of the form $2^{\widetilde{H}(n)}$, we say that a computable trace $\left\{T_{n}\right\}_{n \in \omega}$ is a computable $H$-trace if for each $n$, elements of $T_{n}$ are strictly bounded by $H(n)$. If furthermore $\sum_{n}\left|T_{n}\right| / H(n)$ is finite and computable, the trace $\left\{T_{n}\right\}_{n \in \omega}$ is called a small computable $H$-trace. We say that a function $f<H$ is captured by a computable $H$-trace $\left\{T_{n}\right\}_{n \in \omega}$ if for infinitely many $n$ we have $f(n) \in T_{n}$.

Note that a computable $H$-trace can be small only for functions $H$ such that $\sum_{n} 1 / H(n)<\infty$. The idea underlying small traces is to have $\sum_{n} \lambda\left(\left[T_{n}\right]_{H(n)}\right)$ finite and computable, where $\left[T_{n}\right]_{H(n)}$ is the set of strings of length $\widetilde{H}(n)$ corresponding to elements of $T_{n}$.

We shall see that a sequence is weakly Schnorr engulfing iff it computes some small $H$-trace capturing every computable function bounded by $H$. In order to conduct the proof, the notion of an interval test (see Definition II.7) plays a key role: still via some coding between strings of length $H(n)$ and integers smaller than $H(n)$, we can view any small computable $H$-trace as an interval test, and vice-versa. The goal of this section if to prove the following theorem:

Theorem V.2. A sequence $A$ is weakly Schnorr engulfing iff for some computable function $H$, there is an A-computable small $H$-trace capturing every computable function bounded by $H$.

We start by proving the easy implication of Theorem V.2:

Proposition V.3 (Theorem V. $2 \Leftarrow$ ). If A Turing computes a small $H$-trace capturing every computable function bounded by $H$, then $A$ is weakly Schnorr engulfing.

Proof. Let $H=2^{\widetilde{H}}$ be computable, together with an $A$-computable sequence $\left\{T_{n}\right\}_{n \in \omega}$ of finite sets, such that $\sum_{n}\left|T_{n}\right| / H(n)$ is an $A$-computable real and such that $\left\{T_{n}\right\}_{n \in \omega}$ captures every computable functions bounded by $H$.

We define an interval test $\left\{I_{n}, \mathcal{J}_{n}\right\}_{n \in \omega}$ simply by viewing each member of $T_{n}$ as a string $\sigma$ of length $\widetilde{H}(n)$. Formally let $H^{\prime}(n)=\sum_{m<n} \widetilde{H}(m)$ (with $H^{\prime}(0)=0$ ). Then each $I_{n}$ is equal to $\left[H^{\prime}(n), H^{\prime}(n+1)\right)$ and each $\mathcal{J}_{n}$ is equal to the union of all the sets $[\sigma]_{\left[H^{\prime}(n), H^{\prime}(n+1)\right)}$ for every member of $T_{n}$ encoding a string $\sigma$ of length $\widetilde{H}(n)$.

It is clear that $\left\{I_{n}, \mathcal{J}_{n}\right\}_{n \in \omega}$ is an $A$-computable interval test capturing every computable sequence.

For the converse, the idea is to try to cover any $A$ computable Schnorr test by an $A$-computable interval test. In order to do so we effectivize here some work of Bartoszynski from set theory, but closely related to what we deal with. In particular, some proof of Bartoszynski [2] (see also [3] Theorem 2.5.11) implies that it is not possible in general to cover a Schnorr test by a single interval test ${ }^{2}$. We can however always cover a Schnorr test by two interval tests, the proof we give here being similar to the one of Theorem 2.5.7 of [3]:

Lemma V.4. Given a Schnorr test and a computable sequence of positive rationals $\left\{\varepsilon_{n}\right\}_{n \in \omega}$, there exists a computable sequence of integers $n_{0}<m_{0}<n_{1}<m_{1}<\ldots$ and two computable sequences of clopen sets $\left\{\mathcal{J}_{1, n}\right\}_{n \in \omega}$ and $\left\{\mathcal{J}_{2, n}\right\}_{n \in \omega}$ such that:

- $\mathcal{J}_{1, k} \subseteq 2^{\left[n_{k}, n_{k+1}\right)}$ and $\mathcal{J}_{2, k} \subseteq 2^{\left[m_{k}, m_{k+1}\right)}$

- $\lambda\left(\mathcal{J}_{1, k}\right) \leq \varepsilon_{k}$ and $\lambda\left(\mathcal{J}_{2, k}\right) \leq \varepsilon_{k}$

- Any sequence captured by the Schnorr test is in $\bigcap_{n} \bigcup_{m \geq n} \mathcal{J}_{1, m}$ or in $\bigcap_{n} \bigcup_{m \geq n} \mathcal{J}_{2, m}$.

Furthermore the proposition can be relativized in the obvious way to any oracle $A$.

${ }^{2}$ We don't know if every Schnorr test can be covered by a single independent Schnorr-Solovay test 
Proof. We can suppose without loss of generality that each clopen set of Schnorr-Solovay test consists only of one cylin$\operatorname{der}[\sigma]$. It is then presented by a sequence of stings $\left\{\sigma_{n}\right\}_{n \in \omega}$. We will define an auxiliary computable sequence of integers $p_{0}<q_{0}<p_{1}<q_{1}<\ldots$ such that $\mathcal{J}_{1, k}$ will depend on the strings $\sigma_{i}$ for $q_{k} \leq i<p_{k+1}$. and $\mathcal{J}_{2, k}$ will depend on the strings $\sigma_{i}$ for $p_{k+1} \leq i<q_{k+1}$. The idea is the following: once we've put the first $p$ strings $\sigma_{i}$ in the first component of our first interval test, we remember the maximal length $n$ of those strings, we then put the next $q$ strings in the first component of our second interval test for $q$ sufficiently large such that the sum of the measure of each remaining string is smaller $\varepsilon \times 2^{-n}$. In particular we then know that the sum of the measure of each remaining string to which we remove the $n$ first bits, is still small enough. We then repeat the operation, this time making sure that the measure of what remains to put in the second trace is small enough, and so on, alternating everytime between the two traces. Fig. 2 and Fig. 3 illustrate how this works. Fig. 2 illustrates the choice of $p_{k}<q_{k}<p_{k+1}<q_{k+1}<\ldots$ Fig. 3 illustrates the choice of $n_{k}<m_{k}<n_{k+1}<m_{k+1}<\ldots$.

We now give the formal construction. Let $n_{0}=0$ and $p_{0}=$ 0 . Let $q_{0}>0$ be the least integer such that $\sum_{n \geq q_{0}} 2^{-\left|\sigma_{n}\right|} \leq$ $\varepsilon_{0}$. Let $m_{0}$ be the maximal value between 1 and the length of the longest string $\sigma_{i}$ for $i<q_{0}$. Suppose $p_{k}, n_{k}$ and $q_{k}, m_{k}$ have been defined. Let us define $p_{k+1}$ and $n_{k+1}$. Let $p_{k+1}>$ $q_{k}$ be the least integer such that

$$
\sum_{n \geq p_{k+1}} 2^{-\left|\sigma_{n}\right|} \leq 2^{-m_{k}} \varepsilon_{k}
$$

and $n_{k+1}$ the maximal value between $m_{k}+1$ and the length of the longest string $\sigma_{i}$ for $q_{k} \leq i<p_{k+1}$. Finally let $\mathcal{J}_{1, k}$ be the clopen set equal to the union of $\left[\left.\sigma_{i}\right|_{\left[n_{k}, n_{k+1}\right)}\right]_{\left[n_{k}, n_{k+1}\right)}$ for any $q_{k} \leq i<p_{k+1}$.

Suppose $q_{k}, m_{k}$ and $p_{k+1}, n_{k+1}$ have been defined. Let us define $q_{k+1}$ and $m_{k+1}$. Let $q_{k+1}>p_{k+1}$ be the least integer such that

$$
\sum_{n \geq q_{k+1}} 2^{-\left|\sigma_{n}\right|} \leq 2^{-n_{k+1}} \varepsilon_{k+1}
$$

and let $m_{k+1}$ be the maximal value between $n_{k+1}+1$ and the length of the longest string $\sigma_{i}$ for $p_{k+1} \leq i<q_{k+1}$. Finally let $\mathcal{J}_{2, k}$ be the clopen set equal to the union of $\left[\sigma_{i} \uparrow_{\left[m_{k}, m_{k+1}\right)}\right]_{\left[m_{k}, m_{k+1}\right)}$ for any $p_{k+1} \leq i<q_{k+1}$.

It is clear that $n_{0}<m_{0}<n_{1}<m_{1}<\ldots$ is a computable sequence of integers. It follows that $\mathcal{J}_{1, k} \subseteq 2^{\left[n_{k}, n_{k+1}\right)}$ and $\mathcal{J}_{2, k} \subseteq 2^{\left[m_{k}, m_{k+1}\right)}$ are computable clopen sets, uniformly in $k$. It is also clear that if $\left\{\sigma_{n}\right\}_{n \in \omega}$ contains infinitely many prefixes of $X$, then $X$ is in $\bigcap_{n} \bigcup_{m \geq n} \mathcal{J}_{1, m}$ or in $\bigcap_{n} \bigcup_{m \geq n} \mathcal{J}_{2, m}$. Also note that for any $k$ we have by choice of $q_{k}$ that

$$
\sum_{i \geq q_{k}} 2^{-\left|\sigma_{i}\right|}<2^{-n_{k}} \varepsilon_{k}
$$

Note also that by definition of $\mathcal{J}_{1, k}$ we have

$$
\lambda\left(\mathcal{J}_{1, k}\right) \leq 2^{n_{k}} \sum_{i \geq q_{k}} 2^{-\left|\sigma_{i}\right|}
$$

It follows that $\lambda\left(\mathcal{J}_{1, k}\right) \leq \varepsilon_{k}$. The argument to show $\lambda\left(\mathcal{J}_{2, k}\right) \leq$ $\varepsilon_{k}$ is similar.

In order to obtain Theorem V.2 we would need to merge the two interval tests covering a Schnorr test into a single interval test. As we mentioned it already, Bartoszynski proved that this is not possible. It would be enough, given an $A$ Schnorr test $\bigcap_{n} \mathcal{U}_{n}$ covering every computable sequence, to mix the two interval $A$-Schnorr-Solovay tests obtained with Lemma V.4 into a single interval $A$-Schnorr-Solovay test that also captures every computable sequence, without necessarily covering $\bigcap_{n} \mathcal{U}_{n}$. This is what we achieve now, adapting the proof of Theorem 2.5.12 of [3]. To do so, we need to modify a little bit Lemma V.4, so the sequence $n_{0}<m_{0}<n_{1}<m_{1}<\ldots$ is not just $A$-computable (once we relativize the lemma to $A$ ) but computable. Also in order to achieve this, we need to restrict ourselves to computably dominated oracles $A$. Fortunately this is not a problem to prove Theorem V.2 in full generality. Indeed, we argued already in Proposition II.17 that if $A$ is hyperimmune, it is of i.o.e. degree, and then it certainly computes a small $H$-trace capturing every computable function.

Lemma V.5. Suppose A computably dominated. Given an ASchnorr test and a computable sequence of positive rational $\left\{\varepsilon_{n}\right\}_{n \in \omega}$, there exists a computable sequence of integer $n_{0}<$ $m_{0}<n_{1}<m_{1}<\ldots$ and A-computable sequences of clopen sets $\left\{\mathcal{J}_{1, n}\right\}_{n \in \omega}$ and $\left\{\mathcal{J}_{2, n}\right\}_{n \in \omega}$ such that:

- $\mathcal{J}_{1, k} \subseteq 2^{\left[n_{k}, n_{k+1}\right)}$ and $\mathcal{J}_{2, k} \subseteq 2^{\left[m_{k}, m_{k+1}\right)}$,

- $\lambda\left(\mathcal{J}_{1, k}\right) \leq \varepsilon_{k}$ and $\lambda\left(\mathcal{J}_{2, k}\right) \leq \varepsilon_{k}$

- Any sequence captured by the A-Schnorr test is in $\bigcap_{n} \bigcup_{m \geq n} \mathcal{J}_{1, m}$ or in $\bigcap_{n} \bigcup_{m \geq n} \mathcal{J}_{2, m}$.

Proof. The proof is very similar to the one of Lemma V.4. Let $\left\{\sigma_{n}\right\}_{n \in \omega}$ be a $A$-Schnorr-Solovay test. Similarly we define the sequence of integer $p_{0}<q_{0}<p_{1}<q_{1}<\ldots$ such that $\mathcal{J}_{1, k}$ will depend on the strings $\sigma_{i}$ for $q_{k} \leq i<p_{k+1}$, whereas $\mathcal{J}_{2, k}$ will depend on the strings $\sigma_{i}$ for $p_{k+1} \leq i<q_{k+1}$. The difference is that the sequence $p_{0}<q_{0}<p_{1}<q_{1}<\ldots$ now has to be computable and not just $A$-computable.

To make those sequences computable, we define $F_{A}$ : $\omega \times \mathbb{Q} \rightarrow \omega$ be the $A$-computable function which to $(p, \varepsilon)$ associates the least integer $q>p$ such that $\sum_{n \geq q} 2^{-\left|\sigma_{n}\right|} \leq \varepsilon$. Note that $F_{A}$ is $A$-computable because $\sum_{n} 2^{-\left|\sigma_{n}\right|}$ is $A$ computable. We also define $G_{A}: \omega \times \omega \times \omega \rightarrow \omega$ be the $A$ computable function which to $(p, q, n)$ associates the maximal value between $n+1$ and the length of the longest string $\sigma_{i}$ for $p \leq i<q$. As $A$ is computably dominated, both $F_{A}$ and $G_{A}$ are bounded by some computable functions $F$ and $G$.

Let $n_{0}=0$ and $p_{0}=0$. Let $q_{0}$ be the result of computing $F\left(0, \varepsilon_{0}\right)$. Let $m_{0}$ be the result of computing 


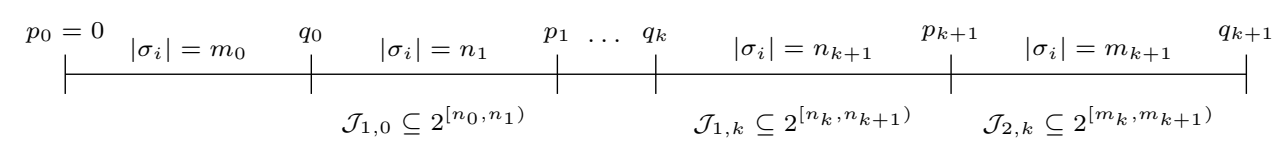

The strings $\sigma_{i}$ for $q_{k} \leq i<p_{k+1}$ are of length $n_{k+1}$

The strings $\sigma_{i}$ for $p_{k+1} \leq i<q_{k+1}$ are of length $m_{k+1}$

Fig. 2. The choice of $p_{k}<q_{k}<p_{k+1}<q_{k+1}<\ldots$

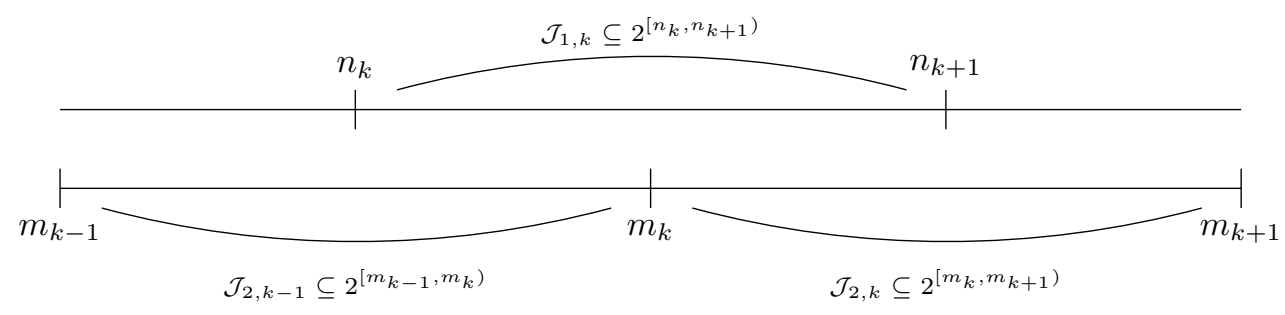

Fig. 3. The choice of $n_{k}<m_{k}<n_{k+1}<m_{k+1}<\ldots$

$G\left(0, q_{0}, 0\right)$. Suppose $p_{k}, n_{k}$ and $q_{k}, m_{k}$ have been defined. Let us define $p_{k+1}$ and $n_{k+1}$. Let $p_{k+1}$ be the result of computing $F\left(q_{k}, 2^{-m_{k}} \varepsilon_{k}\right)$ and $n_{k+1}$ the result of computing $G\left(q_{k}, p_{k+1}, m_{k}\right)$. Finally let $\mathcal{J}_{1, k}$ be the clopen set equal to the union of $\left[\sigma_{i}\left\lceil_{\left[n_{k}, n_{k+1}\right)}\right]_{\left[n_{k}, n_{k+1}\right)}\right.$ for any $q_{k} \leq i<p_{k+1}$.

Suppose $q_{k}, m_{k}$ and $p_{k+1}, n_{k+1}$ have been defined. Let us define $q_{k+1}$ and $m_{k+1}$. Let $q_{k+1}$ be the result of computing $F\left(p_{k+1}, 2^{-n_{k+1}} \varepsilon_{k+1}\right)$ and let $m_{k+1}$ be the result of computing $G\left(p_{k+1}, q_{k+1}, n_{k+1}\right)$. Finally let $\mathcal{J}_{2, k}$ be the clopen set equal to the union of $\left[\sigma_{i} \uparrow_{\left[m_{k}, m_{k+1}\right)}\right]_{\left[m_{k}, m_{k+1}\right)}$ for any $p_{k+1} \leq i<$ $q_{k+1}$.

It is clear that $n_{0}<m_{0}<n_{1}<m_{1}<\ldots$ is a computable sequence of integers. It follows that $\mathcal{J}_{1, k} \subseteq 2^{\left[n_{k}, n_{k+1}\right)}$ and $\mathcal{J}_{2, k} \subseteq 2^{\left[m_{k}, m_{k+1}\right)}$ are $A$-computable clopen sets, uniformly in $k$. The rest of the verification is as in Lemma V.4.

We are now ready to mix the two interval $A$-SchnorrSolovay tests into one interval $A$-Schnorr-Solovay test capturing every computable sequence:

Theorem V.6. Let $A$ be weakly Schnorr engulfing. There exists $\left\{I_{n}, \mathcal{J}_{n}\right\}_{n \in \omega}$ interval tests relative to $A$ capturing every computable sequence. Moreover, the sequence $\left\{I_{n}\right\}_{n \in \omega}$ is computable.

Proof. We already argued the case $A$ not computably dominated. We can then suppose $A$ computably dominated. Fix a decreasing computable sequence of positive rationals $\left\{\varepsilon_{n}\right\}_{n \in \omega}$ such that $\sum_{n} \varepsilon_{n} \times 2^{n+1}<\infty$. By the previous lemma, we can assume that we have a computable sequence of integers $n_{0}<m_{0}<n_{1}<m_{1}<\ldots$, together with two $A$-computable sequences of clopen sets $\left\{\mathcal{J}_{1, n}\right\}_{n \in \omega}$ and
$\left\{\mathcal{J}_{2, n}\right\}_{n \in \omega}$ such that:

- $\mathcal{J}_{1, k} \subseteq 2^{\left[n_{k}, n_{k+1}\right)}$ and $\mathcal{J}_{2, k} \subseteq 2^{\left[m_{k}, m_{k+1}\right)}$

- $\lambda\left(\mathcal{J}_{1, k}\right) \leq \varepsilon_{k}$ and $\lambda\left(\mathcal{J}_{2, k}\right) \leq \varepsilon_{k}$

- Any computable sequence is in $\bigcap_{n} \bigcup_{m \geq n} \mathcal{J}_{1, m}$ or in $\bigcap_{n} \cup_{m \geq n} \mathcal{J}_{2, m}$

We are going to create relative to $A$ an interval test $\left\{\left[n_{k+1}, n_{k+2}\right), \mathcal{R}_{k}\right\}_{k \in \omega}$ by mixing $\left\{\mathcal{J}_{1, k}\right\}_{k \in \omega}$ and $\left\{\mathcal{J}_{2, k}\right\}_{k \in \omega}$. Then assuming that a computable sequence $X$ is not in $\bigcap_{n} \bigcup_{m>n} \mathcal{R}_{m}$ we are going to create, using $X$, another interval $A$-Schnorr-Solovay test $\left\{\left[m_{k+1}, n_{k+2}\right), \mathcal{T}_{k}\right\}_{k \in \omega}$ such that every computable sequence is necessarily in $\bigcap_{n} \cup_{m \geq n} \mathcal{T}_{m}$. To help the understanding of the construction, the reader can refer to Fig. 4.

For $k>0$, Let $\mathcal{S}_{1, k}$ be the union of $[\sigma]_{\left[n_{k}, m_{k}\right)}$ for every strings $\sigma$ of length $m_{k}-n_{k}$ such that there are at least $2^{n_{k+1}-m_{k}-k}$ many strings $\tau$ with $\left[\sigma^{\wedge} \tau\right]_{\left[n_{k}, n_{k+1}\right)} \subseteq \mathcal{J}_{1, k}$. We have $\lambda\left(\left[S_{1, k}\right]^{\prec}\right) 2^{-k} \leq \lambda\left(\mathcal{J}_{1, k}\right)$ and then $\lambda\left(\left[S_{1, k}\right]^{\prec}\right) \leq 2^{k} \varepsilon_{k} \leq$ $2^{k} \varepsilon_{k-1}$.

For $k>0$, let $\mathcal{S}_{2, k}$ be the union of $[\sigma]_{\left[n_{k}, m_{k}\right)}$ for every string $\sigma$ of length $m_{k}-n_{k}$ such that there are at least $2^{n_{k}-m_{k-1}-k}$ many strings $\tau$ with $\left[\tau^{\wedge} \sigma\right]_{\left[m_{k-1}, m_{k}\right)} \subseteq \mathcal{J}_{2, k-1}$. We have $\lambda\left(\left[S_{2, k}\right]^{\prec}\right) 2^{-k} \leq \lambda\left(\mathcal{J}_{2, k-1}\right)$ and then $\lambda\left(\left[S_{2, k}\right]^{\prec}\right) \leq$ $2^{k} \varepsilon_{k-1}$.

Let $\mathcal{R}_{k}=\left[S_{1, k+1}\right]^{\prec} \cup\left[S_{2, k+1}\right]^{\prec}$. By the choice of the $\varepsilon_{k}$ we clearly have that $\left\{\left[n_{k+1}, n_{k+2}\right), \mathcal{R}_{k}\right\}_{k \in \omega}$ is an interval $A$ Schnorr-Solovay test. If every computable sequence belongs to $\bigcap_{n} \cup_{m \geq n} \mathcal{R}_{m}$ we are done. Otherwise there is a computable sequence $X$ such that $X \uparrow_{\left[n_{k+1}, n_{k+2}\right)}$ never belongs to $\mathcal{R}_{k}$ for any $k$.

For $k>0$ let $\mathcal{T}_{1, k}$ be the union of $[\tau]_{\left[m_{k}, n_{k+1}\right)}$ for any string $\tau$ of length $n_{k+1}-m_{k}$ such that $\left[X \uparrow_{\left[n_{k}, m_{k}\right)}{ }^{\wedge} \tau\right]_{\left[n_{k}, n_{k+1}\right)} \subseteq$ 


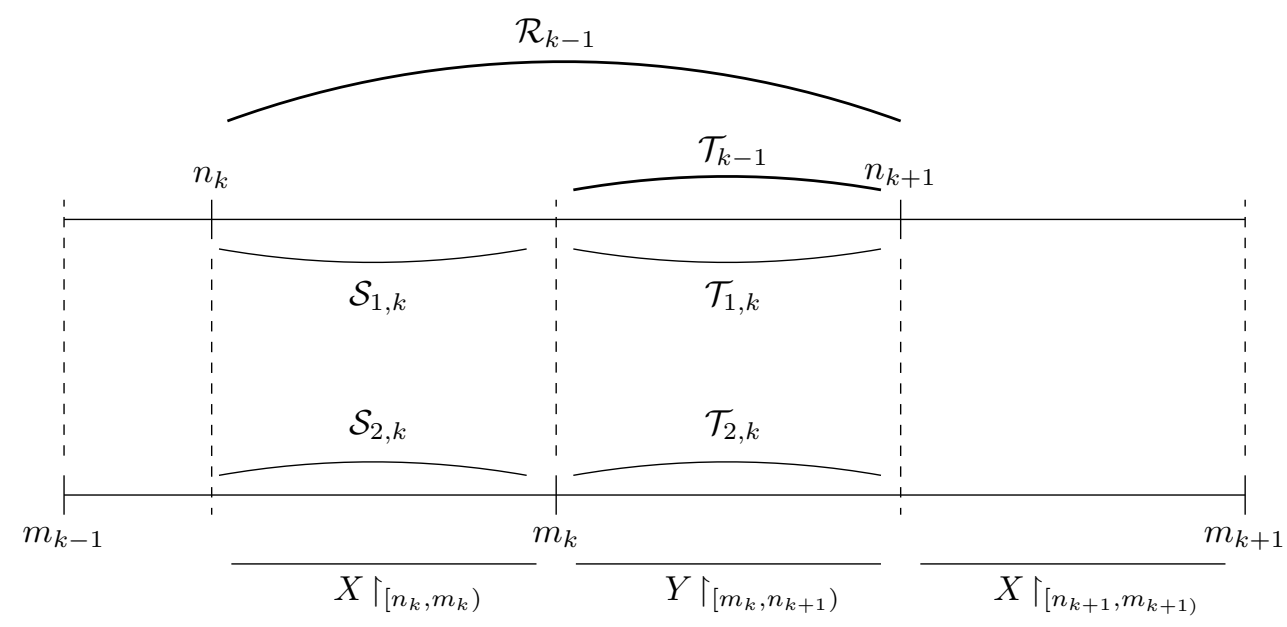

Fig. 4. Construction for Theorem V.6

$\mathcal{J}_{1, k}$ and let $\mathcal{T}_{2, k}$ be the union of $[\tau]_{\left[m_{k}, n_{k+1}\right)}$ for any string $\tau$ of length $n_{k+1}-m_{k}$ such that $\left[\tau^{\wedge} X \uparrow_{\left[n_{k+1}, m_{k+1}\right)}\right]_{\left[m_{k}, m_{k+1}\right)} \subseteq$ $\mathcal{J}_{2, k}$.

As $X$ must be in $\bigcap_{n} \bigcup_{m \geq n} \mathcal{J}_{1, m}$ or in $\bigcap_{n} \bigcup_{m \geq n} \mathcal{J}_{2, m}$ and as no prefix of $X$ belongs to $\mathcal{R}_{k}$ for any $k$, it implies by definition of $\mathcal{S}_{1, k}$ that there are at most $2^{n_{k+1}-m_{k}-k}$ many $[\tau]_{\left[m_{k}, n_{k+1}\right)}$ in $T_{1, k}$ and by definition of $\mathcal{S}_{2, k+1}$ that there are at most $2^{n_{k+1}-m_{k}-k-1}$ many $[\tau]_{\left[m_{k}, n_{k+1}\right)}$ in $T_{2, k}$.

In particular the clopen set $\mathcal{T}_{k}$ defined to be $\mathcal{T}_{1, k+1} \cup$ $\mathcal{T}_{2, k+1}$, has measure smaller than $2^{-k+1}$. Also the set $\left\{\left[m_{k+1}, n_{k+2}\right), \mathcal{T}_{k}\right\}_{k \in \omega}$ is an $A$-interval test.

We now use the fact that the sequence $n_{0}<m_{0}<$ $n_{1}<m_{1}<\ldots$ is computable in order to prove that $\bigcap_{n} \bigcup_{m \geq n} \mathcal{T}_{m}$ contains every computable sequence. Suppose otherwise, that is, some computable sequence $Y$ is in no set $\mathcal{T}_{k}$ for any $k$. Then the sequence $Z=X \uparrow_{\left[n_{0}, m_{0}\right)}{ }^{\wedge} Y \uparrow_{\left[m_{0}, n_{1}\right)}$ ${ }^{\wedge} \ldots{ }^{\wedge} X \uparrow_{\left[n_{k}, m_{k}\right)}{ }^{\wedge} Y \uparrow_{\left[m_{k}, n_{k+1}\right)}$ is computable. Also we easily see that $Z$ is not in $\bigcap_{n} \bigcup_{m \geq n} \mathcal{J}_{1, m}$ as otherwise by definition of $\mathcal{T}_{1, k}$ the sequence $\bar{Y}$ would be captured by $\left\{\left[m_{k+1}, n_{k+2}\right), \mathcal{T}_{k}\right\}_{k \in \omega}$. Similarly we see that $Z$ is not in $\bigcap_{n} \bigcup_{m>n} \mathcal{J}_{2, m}$ as otherwise by definition of $\mathcal{T}_{2, k}$ the sequence $Y$ would be captured $\left\{\left[m_{k+1}, n_{k+2}\right), \mathcal{T}_{k}\right\}_{k \in \omega}$. This then contradicts that $\bigcap_{n} \bigcup_{m \geq n} \mathcal{T}_{m}$ does not contain every computable sequence.

Finally we can prove the hard direction of Theorem V.2:

Theorem V.2 $\Rightarrow$. Given a sequence $A$ weakly Schnorr engulfing, we have an interval $A$-Schnorr-Solovay test $\left\{I_{n}, \mathcal{J}_{n}\right\}_{n \in \omega}$ capturing every computable sequence with in addition that $\left\{I_{n}\right\}_{n \in \omega}$ is computable. We define the computable function $H(n)=2^{\left|I_{n}\right|}$ and the small $A$-computable $H$-trace $\left\{T_{n}\right\}_{n \in \omega}$ to be the set of each integer encoded by each strings $\sigma$ such that $[\sigma]_{I_{n}} \subseteq \mathcal{J}_{n}$. Suppose that a computable function $f: \omega \rightarrow \omega$ bounded by $H$ is not captured by $\left\{T_{n}\right\}_{n \in \omega}$, then also the computable sequence $X$ such that $X \uparrow_{I_{n}}$ is equal to the string of length $\left|I_{n}\right|$ corresponding to $f(n)$ and such that
$X(i)=0$ for $i$ not in any $I_{n}$, is not captured by $\left\{I_{n}, \mathcal{J}_{n}\right\}_{n \in \omega}$ which is a contradiction.

We end the section with an interesting curiosity: In some sense, among the weakly Schnorr engulfing sequences, those which are computably dominated have more capturing power than those which are not. Recall that a sequence is $\Delta_{2}^{0}$ iff it is computable from the halting problem, $\emptyset^{\prime}$, iff it is the pointwise limit of a uniform list of computable sequences.

Proposition V.7. Suppose $A$ is a computably dominated, weakly Schnorr engulfing oracle. Then the set of $\Delta_{2}^{0}$ sequences is contained in an A-Schnorr null set.

Proof. We shall prove that any $A$-Schnorr-Solovay test $\left\{I_{n}, \mathcal{J}_{n}\right\}_{n \in \omega}$, with each $I_{n}$ computable uniformly in $n$, capturing every computable sequence, also captures every $\Delta_{2}^{0}$ sequence. Consider a $\Delta_{2}^{0}$ sequence $X$, given as the pointwise limit of a list $\left\{X_{s}\right\}_{s \in \omega}$ where each $X_{s} \in 2^{\omega}$ is computable uniformly in $s$.

Suppose $X$ is not captured by our $A$-Schnorr-Solovay test, without loss of generality we can suppose that for every $n$ we have $X\left\lceil_{I_{n}} \notin \mathcal{J}_{n}\right.$. Let us define the $A$-computable function $f$ which to the pair $(t, n)$ associate the smallest $s \geq t$ such that $X_{s} \uparrow_{I_{n}} \notin \mathcal{J}_{n}$. As $A$ is computably dominated, the function $f$ is bounded by some computable function $g: \omega \times \omega \mapsto \omega$.

We then define a computable sequence $Y$ such that $Y \uparrow_{I_{n}} \notin$ $\mathcal{J}_{n}$ for each $n$. Fix some $n$ and compute successive values of $g(s, n)$ for every $s$ starting from 0 , until every value $X_{t} \uparrow_{I_{n}}$ is identical for $s \leq t \leq g(s, n)$. As the sequence $\left\{X_{t} \uparrow_{I_{n}}\right\}_{t \in \omega}$ converges, we know that this will happen eventually. Define then $Y \uparrow_{I_{n}}$ to be $X_{g(s, n)} \uparrow_{I_{n}}$ and $Y$ be 0 on positions which are in no $I_{n}$.

It is then clear that $Y \uparrow_{I_{n}} \notin \mathcal{J}_{n}$ for every $n$, because we have $X_{f(s, n)} \uparrow_{I_{n}} \notin \mathcal{J}_{n}$ and as $s \leq f(s, n) \leq g(s, n)$ we have by the choice of $s$ that $X_{g(s, n)}\left\lceil_{I_{n}}=X_{f(s, n)} \Upsilon_{I_{n}}\right.$.

Proposition V.7 does not work if $A$ is hyperimmune. We can simply with $A=\emptyset^{\prime}$ consider the fact that no $A$-Schnorr test 
contains every $A$-computable element. It is worth mentioning that the following analogue of Proposition V.7 is true with a similar proof: If $f$ is computably dominated and $H$-i.o.e., then also $f$ is equal infinitely often to every $\Delta_{2}^{0}$ function bounded by $H$.

\section{ACKNOWLEDGMENT}

The authors would like to thank the Marsden fund of New Zealand, as well as the Science Faculty of the University of Auckland for supporting Monin's research stay in Auckland.

\section{REFERENCES}

[1] U. Andrews, M. Cai, D. Diamondstone, C. Jockush, and S. Lempp, "Asymptotic density, computable traceability, and 1-randomness," 2013.

[2] T. Bartoszyński, "On covering of real line by null sets," Pacific Journal of Mathematics, vol. 131, no. 1, pp. 1-12, 1988.

[3] T. Bartoszyński and H. Judah, Set Theory. On the structure of the real line. Wellesley, MA: A K Peters, 1995, 546 pages.

[4] C. T. Chong, A. Nies, and L. Yu, "Lowness of higher randomness notions," Israel journal of mathematics, vol. 66, no. 1, pp. 39-60, 2008.

[5] C. T. Chong and L. Yu, "Randomness in the higher setting."

[6] O. Demuth, "Remarks on the structure of tt-degrees based on constructive measure theory," Comment. Math. Univ. Carolin., vol. 29, no. 2, pp. 233-247, 1988.

[7] R. Downey and E. Griffiths, "Schnorr randomness," J. Symbolic Logic, vol. 69, no. 2, pp. 533-554, 2004.

[8] R. Downey and D. Hirschfeldt, Algorithmic randomness and complexity. Berlin: Springer-Verlag, 2010, 855 pages.

[9] D. R. Hirschfeldt, C. G. Jockusch Jr, T. McNicholl, and P. E. Schupp, "Asymptotic density and the coarse computability bound," In preparation.

[10] C. G. Jockusch and P. E. Schupp, "Generic computability, turing degrees, and asymptotic density," Journal of the London Mathematical Society, vol. 85, no. 2, pp. 472-490, 2012.

[11] I. Kapovich, A. Myasnikov, P. Schupp, and V. Shpilrain, "Generic-case complexity, decision problems in group theory, and random walks," Journal of Algebra, vol. 264, no. 2, pp. 665-694, 2003.

[12] T. Kihara, "Higher randomness and lim-sup forcing within and beyond hyperarithmetic," In preparation.

[13] B. Kjos-Hanssen, A. Nies, and F. Stephan, "Lowness for the class of Schnorr random sets," SIAM J. Computing, vol. 35, no. 3, pp. 647-657, 2005.

[14] M. Kummer, “A proof of Beigel's cardinality conjecture,” J. Symbolic Logic, vol. 57, no. 2, pp. 677-681, 1992.

[15] S. Kurtz, "Randomness and genericity in the degrees of unsolvability," Ph.D. Dissertation, University of Illinois, Urbana, 1981.

[16] P. Martin-Löf, "The definition of random sequences," Inform. and Control, vol. 9, pp. 602-619, 1966.

[17] B. Monin, "Higher computability and randomness," Ph.D. dissertation, Université Paris Diderot, http://www.liafa.univ-paris-diderot.fr/ $\sim$ benoitm/ressources/misc/Thesis_report_benoit_monin_v1.7.pdf, 2014.

[18] A. Nies, Computability and randomness, ser. Oxford Logic Guides. Oxford: Oxford University Press, 2009, vol. 51. [Online]. Available: http://dx.doi.org/10.1093/acprof:oso/9780199230761.001.0001

[19] J. C. Owings, “A cardinality version of Beigel's Nonspeedup Theorem," J. Symbolic Logic, vol. 54, no. 3, pp. 761-767, 1989.

[20] N. Rupprecht, "Relativized Schnorr tests with universal behavior," Arch. Math. Logic, vol. 49, no. 5, pp. 555-570, 2010. [Online]. Available: http://dx.doi.org.ezproxy.auckland.ac.nz/10.1007/s00153-010-0187-6

[21] G. Sacks, Higher Recursion Theory, ser. Perspectives in Mathematical Logic. Heidelberg: Springer-Verlag, 1990.

[22] C. Schnorr, Zufälligkeit und Wahrscheinlichkeit. Eine algorithmische Begründung der Wahrscheinlichkeitstheorie. Berlin: Springer-Verlag, 1971, lecture Notes in Mathematics, Vol. 218.

[23] S. Terwijn and D. Zambella, "Algorithmic randomness and lowness," $J$. Symbolic Logic, vol. 66, pp. 1199-1205, 2001. 